\title{
Damage Detection of Composite Plates by Lamb Wave Ultrasonic Tomography with a Sparse Hexagonal Network Using Damage Progression Trends
}

\author{
C. J. Keulen, ${ }^{1}$ M. Yildiz, ${ }^{2}$ and A. Suleman ${ }^{1}$ \\ ${ }^{1}$ Department of Mechanical Engineering, University of Victoria, Victoria, BC, Canada V8W 3P6 \\ ${ }^{2}$ Faculty of Engineering and Natural Sciences, Sabanci University, Orhanli-Tuzla, 34956 Istanbul, Turkey \\ Correspondence should be addressed to A. Suleman; suleman@uvic.ca
}

Received 24 July 2013; Accepted 15 November 2013; Published 4 March 2014

Academic Editor: Gyuhae Park

Copyright @ 2014 C. J. Keulen et al. This is an open access article distributed under the Creative Commons Attribution License, which permits unrestricted use, distribution, and reproduction in any medium, provided the original work is properly cited.

\begin{abstract}
Lamb wave based structural health monitoring shows a lot of potential for damage detection of composite structures. However, currently there is no agreement upon optimal network arrangement or detection algorithm. The objective of this research is to develop a sparse network that can be expanded to detect damage over a large area. To achieve this, a novel technique based on damage progression history has been developed. This technique gives an amplification factor to data along actuator-sensor paths that show a steady reduction in transmitted power as induced damage progresses and is implemented with the reconstruction algorithm for probabilistic inspection of damage (RAPID) technique. Two damage metrics are used with the algorithm and a comparison is made to the more commonly used signal difference coefficient (SDC) metric. Best case results show that damage is detected within $12 \mathrm{~mm}$. The algorithm is also run on a more sparse network with no damage detection, therefore indicating that the selected arrangement is the most sparse arrangement with this configuration.
\end{abstract}

\section{Introduction}

To achieve lighter aerospace structures, damage is allowed to exist during operation as long as it is within safe, predetermined specifications; aircraft structures are designed according to a damage tolerant philosophy. In more recent years composite materials are being used to build aerospace structures because they are lightweight and stiff and have excellent fatigue and corrosion resistance. The downside to composites, however, lies in their damage mechanisms. Composites may fail or become damaged in a number of ways that are very different from traditional metallic materials. Defects may arise during manufacture due to voids/porosity, ply misalignment, or inclusion of foreign objects that show no evidence to the naked eye. Composites suffer from low velocity impacts that can damage the internal structure of a laminate while leaving no visible evidence on the surface.

Maintenance and inspection of aircraft is of utmost importance for safe and efficient operation. Aircraft structures operate in harsh conditions sustaining high loads, fatigue cycles, and extreme temperature differentials. Failure of these structures is not acceptable due to the possibility of loss of life and assets. To ensure aircraft structures are in safe operational condition, costly inspection involving aircraft downtime and often disassembly of major components is routinely performed. The cost of inspection is about $30 \%$ of the total cost of acquiring and operating composite structures [1]. Currently, damage detection is performed with techniques referred to as nondestructive testing (NDT) or nondestructive inspection (NDI) to locate and quantify damage.

To reduce operational cost and improve reliability and performance, a common goal of researchers, designers, and manufacturers is to develop a real time inspection system that is permanently installed or embedded within the structure. This technique is commonly referred to as structural health monitoring (SMH) $[2,3]$. Such systems typically consist of a network of transducers that are used to sense physical parameters that indicate the presence of damage with an interrogation technique or algorithm. 
In 1917, Lamb published his classic analysis and description of acoustic waves, which included the first consideration of Lamb waves [4]. In 1961, Worlton [5] proposed the use of Lamb waves for damage detection and a new NDE potential emerged. In 1962, Frederick and Worlont [6] conducted the first experimental study. In the beginning of the 1990s Hutchins et al. [7-9] and Nagata et al. [10] applied Lamb waves to NDE using medical imaging and seismic tomography techniques to both metallic and composite materials. Their systems utilized pairs of transducers that were positioned across the material at various locations in order to obtain a dense collection of pitch-catch signals in order to reconstruct a tomogram. While the techniques produced reasonable results the methods were time consuming, requiring a lot of repositioning of the transducers, and in some cases requiring the specimen to be placed in a water bath. Prasad et al. [11] implemented an SHM system for composites using surface bonded piezoelectric transducers. Since the transducers were permanently bonded in place the system could be operated in real time without any repositioning. Gao et al. [12] introduced the reconstruction algorithm for probabilistic inspection of damage (RAPID). Michaels [13] later investigated the application of tomography algorithms to sparse networks.

Other research efforts were made to use the Lamb wave mode propagation characteristics in order to detect damage, specifically the attenuation and arrival time of the first two wave modes. In 1993 Guo and Cawley [14] studied the interaction of Lamb waves with delaminations in composite materials both numerically and experimentally. Keilers and Chang [15] later proposed a built-in damage detection system using an array of piezoelectric transducers. Giurgiutiu et al. [16] discussed the pulse-echo analysis technique and subtracting baseline data from damaged data to detect damage.

This paper presents the details and results of a study on the implementation of a sparse piezoelectric transducer network for damage detection in composite materials. The objective of this research is to develop a sparse network that can be expanded to detect damage over a large area. To achieve this, a novel technique based on damage progression history has been developed.

\section{Theory and Implementation}

Lamb waves are elastic, guided waves that propagate parallel to the surface in thin structures with free boundaries. Plates are the best example; however, Lamb waves can also propagate in structures with a shallow curvature. The most advantageous characteristics of these waves are their susceptibility to interferences caused by damages or boundaries (the features of interest) and low amplitude loss. To implement a Lamb wave based damage detection technique some important properties must be determined. When Lamb waves propagate they travel in one of two possible ways with respect to the plate's mid plane. If the motion is symmetric about the midplane (the peaks and troughs of the waves are in phase) then it is a symmetric mode and if the motion is not symmetric (the peaks and troughs are $180^{\circ}$ out of phase) it is an antisymmetric mode. An infinite number of modes exist; each mode is referred to as an $A$ mode or $S$ mode if it is antisymmetric or symmetric, respectively, with a subscript indicating its order. For example the lowest order/frequency symmetric mode is referred to as an $S_{0}$ mode while the second lowest order/frequency symmetric mode is referred to as an $A_{1}$ mode. Each mode exists at a different frequency depending on the properties of the material. At lower frequency-thickness values less modes exist. It is advantageous to operate in a frequency-thickness range where only the $S_{0}$ and $A_{0}$ modes exist. This is generally below $1.5 \mathrm{MHz} \mathrm{mm}$.

2.1. Implementation. To employ Lamb waves for damage detection, a system to send and receive Lamb waves must be developed and a number of parameters must be selected to tune the system such as the transducer type, size and arrangement, actuation signal, and data acquisition.

Piezoelectric transducers are commonly used in SHM systems. They are inherently simple devices consisting of simply a piezoelectric material with two conductive surfaces. When a voltage is applied across the surfaces the material expands or contracts (depending on polarity) proportional to the magnitude of voltage applied. Conversely, when the material is deformed a voltage difference is seen between the surfaces. This property leads to the greatest benefit of piezoelectric transducers: their ability to both send and receive signals. Piezoelectric transducers are small and light and can be bonded to or embedded within a structure with little effect. Signals are voltage based and therefore easy to generate and acquire with common hardware. Lead zirconate titanate (PZT) is the most commonly used material in piezoelectric transducers. They offer excellent performance for both generation and acquisition, have excellent mechanical strength, wide frequency responses, and low power consumption, and can be obtained at a low cost [17].

When actuating Lamb waves it is desirable to actuate the least number of modes possible (preferably only the $S_{0}$ and $A_{0}$ ) so that signal interpretation is simplified. As mentioned earlier, this usually occurs below $1.5 \mathrm{MHz} \mathrm{mm}$. This range is also beneficial because there is low dispersion, which means that if the frequency changes as the wave propagates through the material, its velocity will remain relatively constant, therefore simplifying signal interpretation. It is desirable to send out a single pulse so that the propagation of the wave groups can be analyzed. The challenge then lies in producing an instantaneous pulse that can be controlled and actuated at a desired frequency. There is no control over a frequency generated by a simple impulse; therefore a short burst must be emitted. If a simple sine wave composed of a few cycles is emitted then the desired frequency can be actuated; however, the frequency domain of such a signal shows small secondary peaks in the frequency domain that are present at other frequencies. To eliminate these peaks the signal can be modulated with a window function to slowly increase and decrease the magnitude of the signal [18]. A commonly used signal that provides a good compromise between number of cycles and ramp up rate consists of five 


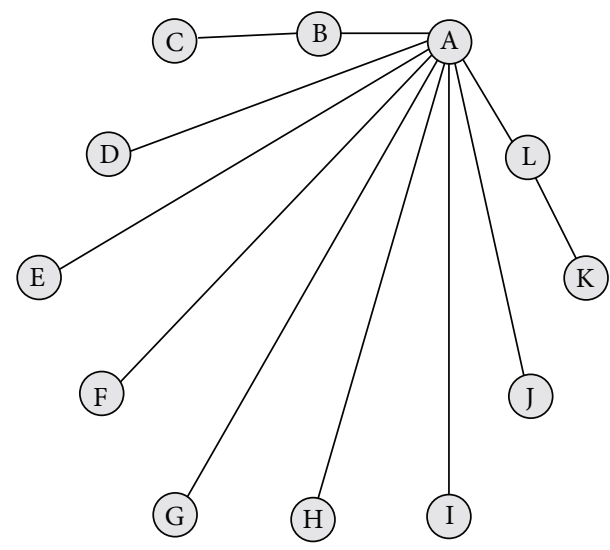

(a)

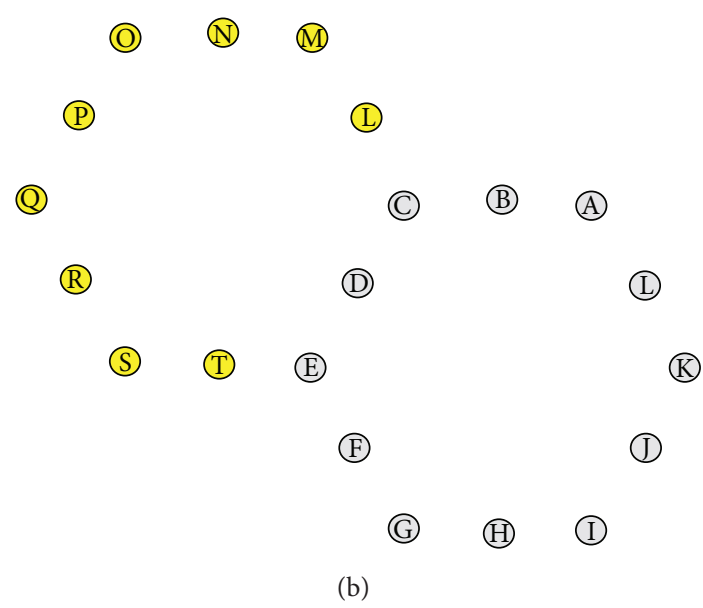

(b)

FIGURE 1: (a) Hexagonal network showing actuator-sensor paths from transducer A (left) and (b) expansion of a single unit cell (right).

sine peaks modulated by a Hann window. A signal with an odd number of peaks is used so that there is a clear maximum peak that can be used for signal processing (if an even number of peaks were used then there would be two peaks with the same maximum amplitude).

2.2. Proposed Network Technique. The technique developed in this research aims to provide a practical, modular network that is sparse and can be expanded to cover a large area. Generally, tomography requires a dense network of transducers that cannot be easily expanded to cover a larger inspection area. The proposed technique makes use of a hexagonal arrangement, which is modular in the sense that it uses a "unit cell" that can be repeated to expand the network to cover a large inspection area. The unit cell consists of 12 transducers in a hexagonal arrangement as shown in Figure 1(a). The network can be expanded by simply increasing the number of unit cells as shown in Figure 1(b). A further benefit is that two unit cells can share three transducers, which means that another unit cell only requires nine new transducers.

Each transducer can act as both an actuator and a sensor. Inspection begins by actuating one transducer to send Lamb waves through the material and recording the signals with the remaining transducers. This is then repeated 11 times such that each transducer acts as an actuator once. Since there is one actuator and 11 sensors, there are 11 actuator-sensor pairs per transducer with direct paths between them as shown in Figure 1(a).

2.3. Damage Location Algorithm. Once data is collected in undamaged and various damaged states, a technique must be implemented to locate the damage. Various techniques have been developed, each relies on a difference between the damaged and undamaged state. Such techniques include delay-and-sum beam-forming [19], the time-difference-ofarrival method [20], the energy arrival method [21], and the filtered back-projection method [22]. To implement the proposed sparse hex network an algorithm was developed that incorporates damage progression information. This algorithm can be incorporated with existing algorithms to increase their accuracy.

The algorithm selected for this research was the reconstruction algorithm for probabilistic inspection of damage (RAPID) [12]. It was developed for networks based on 816 transducers and has inherently good signal-to-meannoise ratios [13]; it can accept various input parameters and produces reasonably accurate results [22]. Changes in the transmitted Lamb wave signal are related to a change in the material properties (i.e., damage) between two sensors. The probability of defect presence at a certain point can be reconstructed from the severity of the signal change and its relative position to the actuator/sensor pair [12]. The RAPID algorithm is based on two assumptions: (i) all effects from every possible actuator/sensor pair can be expressed as a linear summation across the entire inspection region and (ii) information from a specific actuator/sensor pair contributes to the defect distribution estimation of a subregion is the vicinity of the path between the pair.

Equation (1) describes the RAPID algorithm:

$$
P(x, y)=\sum_{k=1}^{N} p_{k}(x, y)=\sum_{k=1}^{N} A_{k}\left(\frac{\beta-R}{\beta-1}\right),
$$

where $P(x, y)$ is the probability of the existence of a defect at position $(x, y)$, the Cartesian coordinate of a point in the inspection area, $A_{k}$ is the damage metric as described below, $\beta$ is a scaling factor that defines the subregion that the actuator/sensor pair has an effect on (essentially an ellipse with the actuator and sensor at its foci), and $R(x, y)$ is described in (2) as

$$
\begin{aligned}
& R\left(x, y, x_{1 k}, y_{1 k}, x_{2 k}, y_{2 k}\right) \\
& =\frac{\sqrt{\left(x-x_{1 k}\right)^{2}+\left(y-y_{1 k}\right)^{2}}+\sqrt{\left(x-x_{2 k}\right)^{2}+\left(y-y_{2 k}\right)^{2}}}{\sqrt{\left(x_{1 k}-x_{2 k}\right)^{2}+\left(y_{1 k}-y_{2 k}\right)^{2}}},
\end{aligned}
$$

where $\left(x_{1 k}, y_{1 k}\right)$ is the Cartesian coordinate of the actuator and $\left(x_{2 k}, y_{2 k}\right)$ is the Cartesian coordinate of the sensor. In this 


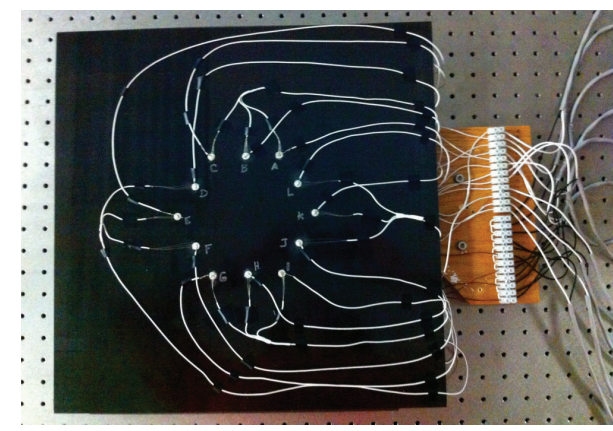

(a)

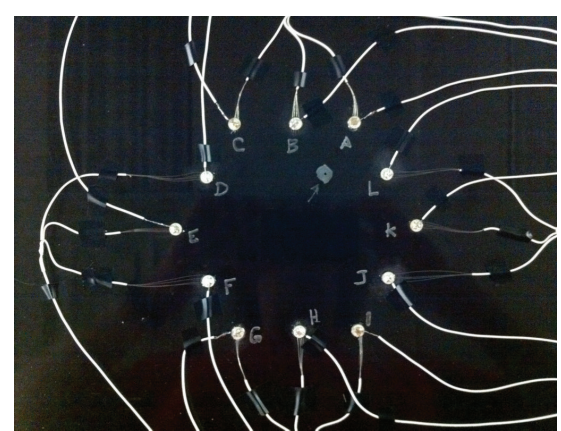

(b)

Figure 2: (a) Composite panel with hex network (left) and (b) hex network with induced damage (right).

work a value of 1.05 was selected for $\beta$ as it is the commonly used value [22-24].

$A_{k}$ is a damage metric that is extracted from the Lamb wave signals. It can be based on various phenomena such as a reduction in transmitted power, reduction in magnitude of waves, or delay in arrival time. More recently Moustafa and Salamone [25] used the fractal dimension with a modified box-counting algorithm. The fractal dimension is a metric used to compare two curves and is calculated as the Hausdorff dimension. The most commonly used metric is the signal difference coefficient (SDC) used by various researchers [2224]. This metric will be used as a baseline to compare results with the proposed algorithm. The signal difference coefficient between two data sets is defined as

$$
\mathrm{SDC}=1-\left|\rho_{a b}\right|,
$$

where

$$
\rho_{a b}=\frac{1}{S} \frac{\sum_{i=1}^{S}\left(a_{i}-\mu_{a}\right)\left(b_{i}-\mu_{b}\right)}{\sqrt{\left\{\sum_{i=1}^{S}\left(a_{i}-\mu_{a}\right)^{2}\right\}}\left\{\sum_{i=1}^{S}\left(b_{i}-\mu_{b}\right)^{2}\right\}}
$$

and $S$ is the total number of samples, $a_{i}$ and $b_{i}$ are the initial data and damaged data, respectively, at sample $i$, and $\mu_{a}$ and $\mu_{b}$ are the arithmetic mean value of the initial data set and damaged data set, respectively.

Most damage detection systems rely solely on detecting damage as it occurs, that is, by comparing the change in the damaged state with the undamaged state. In reality, however, damage often begins as a small flaw that slowly increases in size while in service. As the damage grows, information can be collected that can be used to locate the damage before it is large enough to be detected by algorithms without this information. In this work, a novel technique is developed that incorporates information from the damage progression into the RAPID algorithm to increase the effectiveness and enable its use in sparse networks where it may otherwise not be applicable.

To incorporate damage progression information, the magnitude of power from the transmitted Lamb waves is compared with that from the previous state (the previous hole size in the case of this study). If the power is less then there is the possibility that either damage is progressing in that path or external noise has caused a decrease in the signal. A history of the progression is recorded and if the power consistently drops across a particular path the probability of damage in that location is multiplied by an amplification factor. This is done in order to differentiate between noise and damage progression. Naturally, the initial damage states will not accurately show a damage progression; however, as the trend continues across more damage states the accuracy increases and the results become more reliable. In this study an amplification factor of 1.10 (a 10\% increase) was selected.

\section{Experimental Investigation}

To investigate the potential of the proposed network, experiments were conducted on a composite panel with a single unit cell of 12 transducers arranged in a hexagonal pattern as in Figure 1(a). Reference data was collected while the material was in pristine condition and again with incrementally greater induced damage in the form of a through hole. The data was processed using the RAPID algorithm in order to determine the location of the damage.

3.1. Experimental Setup. A $420 \mathrm{~mm} \times 420 \mathrm{~mm}$ panel, composed of eight plys of $139 \mathrm{gsm}$, unidirectional T700 carbon fiber with West System 105/206 epoxy, was laminated in a $[0 / 90 / \pm 45]_{S}$ orientation to produce a $1.23 \mathrm{~mm}$ thick quasiisotropic composite. A jig was machined to locate and bond $12,1 \mathrm{~mm}$ thick, and $7.56 \mathrm{~mm}$ diameter PZT transducers to the panel in a $75 \mathrm{~mm}$ circumradius hexagonal array. The panel and network are shown in Figure 2(a), while a closeup of the network showing the damage location is shown in Figure 2(b). Each transducer was assigned a letter for reference as seen in Figures 1 and 2. The coordinates of the transducers are as follows: $A$ : $(247.50,274.95), B:(210.00$, 274.95), C: (172.50, 274.95), D: (153.75, 242.48), E: (135.00, 210.00), F: (153.75, 177.53), G: (172.50, 145.05), H: (210.00, 145.05), I: (247.50, 145.05), J: (266.25, 177.53), $K:(285.00$, 210.00), and $L:(266.25,242.48)$ in $\mathrm{mm}$ from the lower left corner of the panel. Damage was induced at $(228.75,241.85)$.

A National Instruments NI PXI-5421 arbitrary waveform generator was used to generate a signal while a NI PXI-5105 digitizer/oscilloscope was used to acquire the signal. Both 


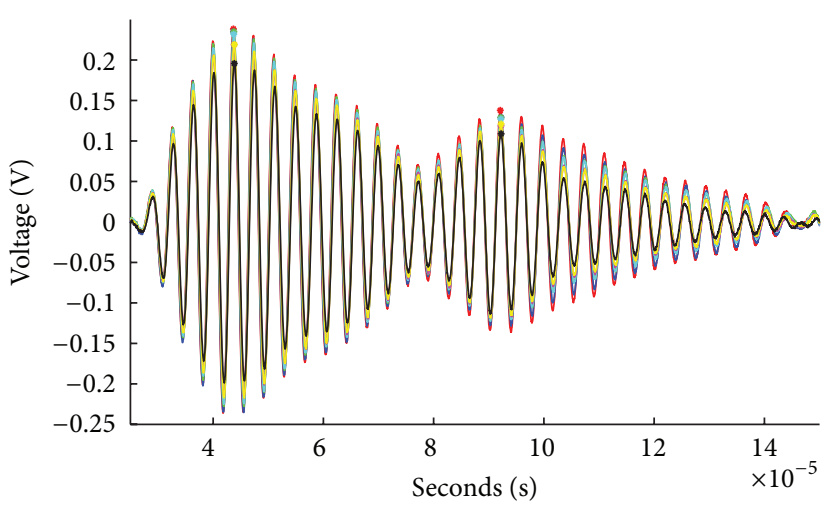

(a)

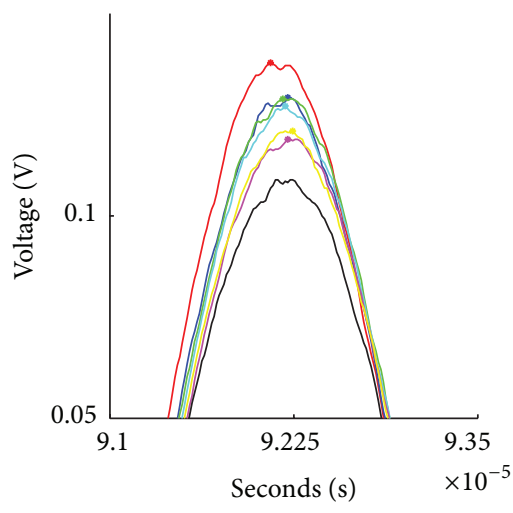

(b)

Figure 3: (a) Recieved Lamb wave signals at all seven damage states along path $A$-G (left) and (b) closeup of $A_{0}$ wave (right).

units were installed in a NI PXI-1033 chassis and controlled with a custom National Instruments LabView program. A model A-303 amplifier from A.A. Labs Ltd. was used to amplify the generated signal.

3.2. Signal Generation and Acquisition. An actuation signal consisting of a sine wave with five peaks modulated by a Hann window was employed. An amplitude of $\pm 8 \mathrm{~V}$ was produced by the waveform generator and amplified by a factor of 20 before it was sent to the actuator resulting in a maximum amplitude of $\pm 160 \mathrm{~V}$. The actuation frequency was selected based on a number of criteria. In order to only actuate the $S_{0}$ and $A_{0}$ modes the frequency-thickness product was kept below $1.5 \mathrm{MHz} \mathrm{mm}$. A frequency scan from $100 \mathrm{kHz}$ to $400 \mathrm{kHz}$ was performed to determine the frequency that transmitted the greatest amplitude. It was found that the frequency range of $260-270 \mathrm{kHz}$ transmitted the greatest amplitude; therefore $265 \mathrm{kHz}$ was used for the experiments.

With these properties selected, the waveform generator was programed to output the actuation signal at $100 \mathrm{MHz}$. Data was acquired at $60 \mathrm{MHz}$ for $30 \times 10^{3}$ samples $(500 \mu \mathrm{s})$. An ASCII text file was written after each run and saved for data processing.

3.3. Experimental Procedure. Once the experimental apparatus was set up as described above, a number of experiments were performed under various damage conditions. Each experiment consists of actuating one transducer and sensing the other eleven, then actuating the adjacent transducer and sensing with the remaining eleven. This process is repeated until all 12 transducers have actuated the system once. At this point the damage is increased and the process is repeated.

Initially the experiment was conducted on the panel in pristine condition before damage was inflicted in the form of a hole drilled through the panel at a location such that it intersected the paths between PZT pairs $A-G$ and $C-K$ as shown in Figure 2(b). The initial diameter of the hole was $1.59 \mathrm{~mm}$, which was increased to $2.38 \mathrm{~mm}, 3.18 \mathrm{~mm}, 4.00 \mathrm{~mm}$, $4.76 \mathrm{~mm}$, and finally $6.35 \mathrm{~mm}$.

\section{Discussion}

The main objective of this work is to develop a modular sparse network. To achieve this an algorithm that uses damage progression information is introduced. With this algorithm, two damage metrics were implemented. The first is based on the SDC in order to compare the proposed algorithm to a conventional metric (SDC). The second damage metric is based on the transmitted power of the signal from an FFT analysis. For both metrics, a comparison between the results with and without the damage progression algorithm is made to assess the effectiveness. A third case was also considered that neglected information from every second sensor, essentially creating a more sparse network of six transducers; however, this did not produce any reasonable results, therefore implying that a minimum of 12 transducers are required in this situation.

Figure 3(a) presents an example of the received $S_{0}$ and $A_{0}$ waveforms actuated by transducer $A$ and received by transducer $G$. All damage states are plotted together on the same chart with their peaks marked with an asterisk $(*)$. Figure 3(b) shows a closeup of the $A_{0}$ peak; a consistent decrease in amplitude due to damage is clearly seen. Signals like these from all paths are processed to determine if a decreasing trend exists.

To benchmark the results, the SDC was calculated for each actuator-sensor path and implemented in the RAPID algorithm. A contour plot indicating the probability of damage is presented in Figure 4(a). The damage location is indicated with the yellow cross and circle. With this information no damage could be detected. This is reasonable according to Michaels [13] who reported that the technique was not highly effective on large, sparse arrays. The aforementioned damage progression information was also implemented with the SDC metric. The results are shown in Figure 4(b). While the algorithm was not able to detect the exact location of the damage it did locate a region close to the general area of the damage, therefore showing a marked increase in accuracy by using damage progression information.

In the second implementation the transmitted power was used as the damage metric. An FFT analysis was performed 


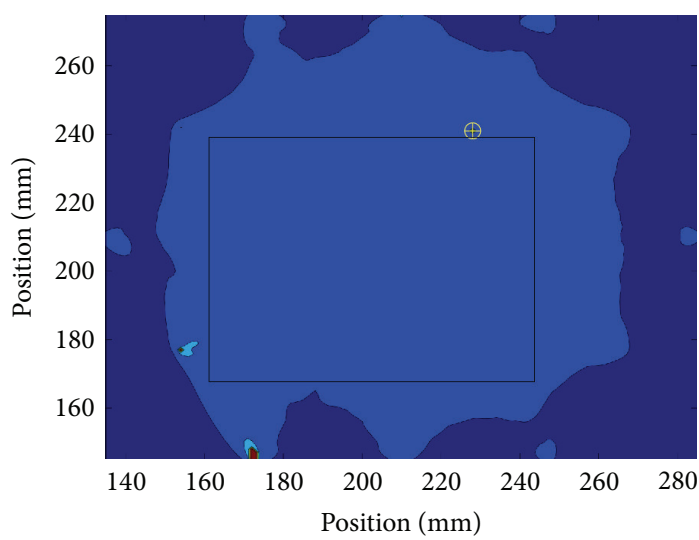

(a)

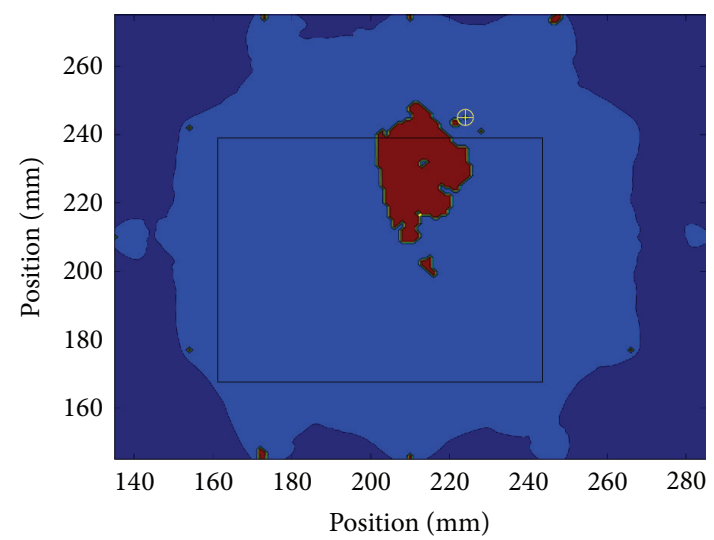

(b)

FIGURE 4: (a) SDC results with no damage progression factor (left) and (b) SDC results with damage progression factor of 1.10 (right) ${ }^{*}$ damage location indicated by yellow cross.

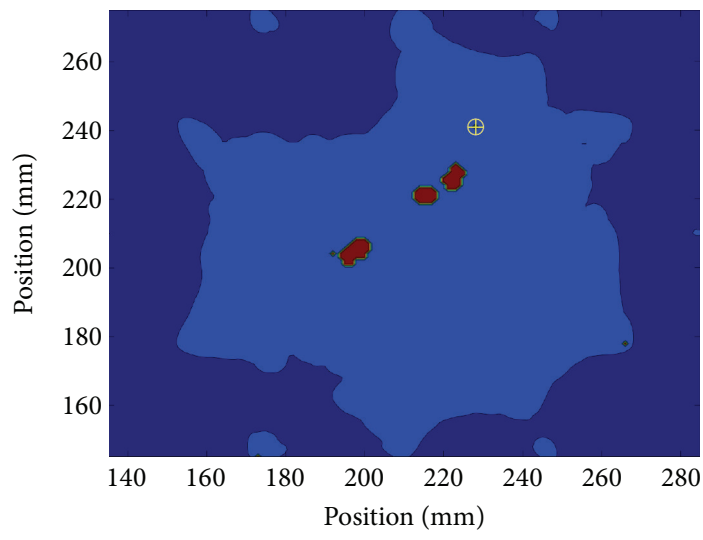

(a)

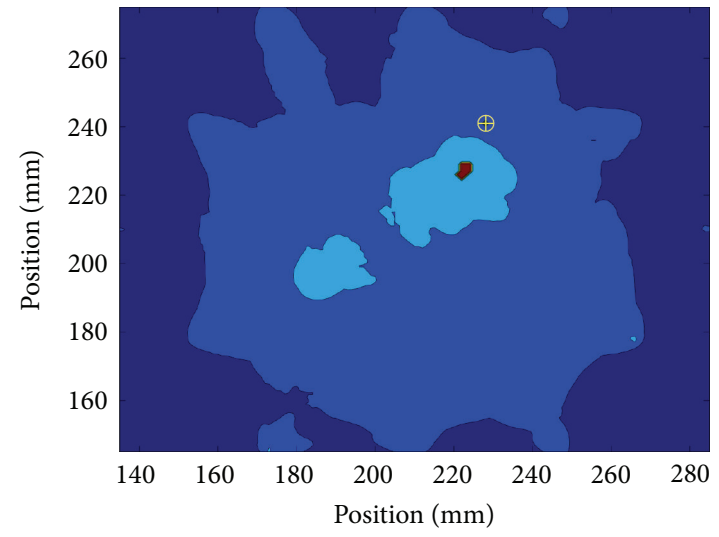

(b)

Figure 5: (a) Power amplitude results with no damage progression factor (left) and (b) power amplitude results with damage progression factor of 1.10 (right) ${ }^{*}$ damage location indicated by yellow cross.

on data from every actuator-sensor path to extract the magnitude of transmitted power. The RAPID algorithm was implemented and the results are shown in Figure 5(a). At this point the algorithm has located three possible damage locations compared to zero locations with the SDC metric. The algorithm was implemented with the damage progression information as shown in Figure 5(b). With this information the algorithm has located a small region of damage roughly the same size within a $12 \mathrm{~mm}$ radius. While these results are not highly accurate they do demonstrate that the use of information from the damage progression does increase the probability of damage detection and allows a large, sparse network to be used.

Many similar research efforts focus on aluminum rather than composite making it difficult to compare results. The results from the experiments performed here are an improvement over the work on an aluminum panel by Hay et al. [22] as a larger spaced array is implemented with fewer transducer with results comparable to Liu et al.s [26] who were able to detect a $30 \mathrm{~mm} \times 30 \mathrm{~mm}$ delamination to within
$10.2 \mathrm{~mm}$ using an array of four transducer pairs arranged in a square with sides of $225 \mathrm{~mm}$ in length. In general, this technique allows for a larger spaced array with a lower density of transducers.

In real world structures, the effects of temperature fluctuation and material anisotropy would have an effect on the signals used in this algorithm and could possibly create inaccurate results. In the case of anisotropy, this would be known a priori as the laminate schedule and material properties of the structure are known. It could be taken into account by normalizing the signals to compare all sensor-actuator pairs if needed. Since this technique relies on signals that travel in a straight line between sensoractuator pair that are compared to the same signal from a previous damage state, the effect of anisotropy would not have an influence because it remains constant (aside from the effects of damage). Temperature, however, can change drastically during service. As the temperature of a carbon fiber/epoxy laminate increases, the modulus of elasticity decreases and therefore the magnitude of the signal that it 
transmits decreases. To address this, a temperature sensor could be used to measure the temperature of the laminate in the region being monitored and with the effect of temperature on the signal transmission known, the effects of temperature could be accounted for in the algorithm.

\section{Conclusion}

A novel sparse network that can be expanded to cover any size area has been proposed along with a novel technique for incorporating information on the progression of damage to improve the accuracy of existing algorithms.

The network consists of a unit cell of 12 piezoelectric transducers arranged in a hexagonal pattern as shown in Figure 1(a). The network is easily expanded by adding nine more transducers to the existing 12 as shown in Figure 1(b) (two unit cells). The hexagonal arrangement allows for efficient stacking of unit cells that would not be otherwise possible with a circular array.

Damage progression information was implemented in the form of a $10 \%$ increase in signal magnitude if the actuatorsensor path showed a consistent decrease in power across each damage state (i.e., if the power was consistently less than the previous state). The results presented in Figure 4 show that incorporating the damage progression information increases the chance of detection with SDC as a damage metric. When the power of the transmitted wave is used as a damage metric some potential damage locations appear as shown in Figure 5(a). When the damage progression information is incorporated the accuracy is increased, locating a potential damage site that is within $12 \mathrm{~mm}$ of the actual damage as shown in Figure 5(b). While the results do not directly pinpoint the damage location they do show that an improvement over the existing RAPID algorithm can be made by incorporating damage progression information with the use of a larger area sparse network on a carbon fiber composite material.

\section{Conflict of Interests}

The authors declare that there is no conflict of interests regarding the publication of this paper.

\section{References}

[1] Y. Bar-Cohen, A. Mal, S.-S. Lih, and Z. Chang, "Composite materials stiff- ness determination and defects characterization using enhanced leaky Lamb wave dispersion data acquisition method," in Proceedings of the SPIE Annual International Symposium on NDE of Aging Aircraft, Airports, and Aerospace Hardware, 1999.

[2] K. Worden, C. R. Farrar, G. Manson, and G. Park, "The fundamental axioms of structural health monitoring," Proceedings of the Royal Society A, vol. 463, no. 2082, pp. 1639-1664, 2007.

[3] K. Worden, "An introduction to structural health monitoring," The Philosophical Transactions of the Royal Society A, vol. 365, no. 1851, pp. 303-315, 2007.

[4] H. Lamb, "On waves in an elastic plate," Proceedings of the Royal Society A, vol. 93, pp. 114-128, 1917.
[5] D. Worlton, "Experimental confirmation of lamb waves at megacycle frequencies," Journal of Applied Physics, vol. 32, no. 6, pp. 967-971, 1961.

[6] C. Frederick and D. Worlont, "Ultrasonic thickness measurements with Lamb waves," Journal of Nondestructive Testing, vol. 20, pp. 51-55, 1962.

[7] D. Jansen and D. Hutchins, "Lamb wave tomography," in Proceedings of the IEEE 1990 Ultrasonics Symposium, pp. 10171020, December 1990.

[8] D. Hutchins, D. Jansen, and C. Edwards, "Lamb-wave tomography using non-contact transduction," Ultrasonics, vol. 31, no. 2, pp. 97-103, 1993.

[9] D. Jansen, D. Hutchins, and J. Mottram, "Lamb wave tomography of advanced composite laminates containing damage," Ultrasonics, vol. 32, no. 2, pp. 83-90, 1994.

[10] Y. Nagata, J. Huang, J. Achenback, and S. Krishnaswamy, "Lamb wave tomography using laser-based ultrasonics," in Review of Progress in Quantitative Nondestrcutive Evaluation, vol. 14, pp. 561-568, 1995.

[11] S. Prasad, K. Balasubramaniam, and C. Krishnamurthy, "Structural health monitoring of composite structures using Lamb wave tomography," Smart Materials and Structures, vol. 13, no. 5, pp. N77-N79, 2004.

[12] H. Gao, Y. Shi, and J. L. Rose, "Guided wave tomography on an aircraft wing with leave in place sensors," in Proceedings of the Review of Progress in Quantitative Nondestructive Evaluation Conference (AIP' 05), pp. 1788-1794, 2005.

[13] J. Michaels, "Effectiveness of in situ damage localization methods using sparse ultrasonic sensor arrays," in Proceedings of the SPIE, March 2008.

[14] N. Guo and P. Cawley, "The interaction of Lamb waves with delaminations in composite laminates," Journal of the Acoustical Society of America, vol. 94, no. 4, pp. 2240-2246, 1993.

[15] C. H. Keilers Jr. and F. K. Chang, "Identifying delamination in composite beams using built-in piezoelectrics: part I-experiments and analysis," Journal of Intelligent Material Systems and Structures, vol. 6, no. 5, pp. 649-663, 1995.

[16] V. Giurgiutiu, A. Zagrai, and J. J. Bao, "Piezoelectric wafer embedded active sensors for aging aircraft structural health monitoring," Structural Health Monitoring, vol. 1, no. 1, pp. 4161, 2002.

[17] Z. Su, L. Ye, and Y. Lu, "Guided Lamb waves for identification of damage in composite structures: a review," Journal of Sound and Vibration, vol. 295, no. 3-5, pp. 753-780, 2006.

[18] B. Rocha, C. Silva, and A. Suleman, "Structural health monitoring system using piezoelectric networks with tuned lamb waves," Shock and Vibration, vol. 17, no. 4-5, pp. 677-695, 2010.

[19] C. Wang, J. Rose, and F. Chang, "A synthetic time-reversal imaging method for structural health monitoring," Smart Materials and Structures, vol. 13, no. 2, pp. 415-423, 2004.

[20] J. Michaels, A. Croxford, and P. Wilcox, "Imaging algorithms for locating damage via in situ ultrasonic sensors," in Proceedings of the 3rd IEEE Sensors Applications Symposium (SAS '08), pp. 6367, February 2008.

[21] J. Michaels and T. Michaels, "Damage localization in inhomogeneous plates using a sparse array of ultrasonic transducers," in AIP Conference Proceedings of the Review of Progress in Quantitative Nondestructive Evaluation, vol. 26, pp. 846-853, August 2006.

[22] T. Hay, R. Royer, H. Gao, X. Zhao, and J. L. Rose, "A comparison of embedded sensor Lamb wave ultrasonic tomography 
approaches for material loss detection," Smart Materials and Structures, vol. 15, no. 4, pp. 946-951, 2006.

[23] J. V. Velsor, H. Gao, and J. Rose, "Guided-wave tomographic imaging of defects in pipe using a probabilistic reconstruction algorithm," Insight, vol. 49, no. 9, pp. 532-537, 2007.

[24] F. Yan, R. Royer Jr., and J. Rose, "Ultrasonic guided wave imaging techniques in structural health monitoring," Journal of Intelligent Material Systems and Structures, vol. 21, no. 3, pp. 377384, 2010.

[25] A. Moustafa and S. Salamone, "Fractal dimension-based Lamb wave tomog- raphy algorithm for damage detection in platelike structures," Journal of Intelligent Material Systems and Structures, vol. 23, no. 11, pp. 1269-1276, 2012.

[26] Y. Liu, M. Fard, A. Chattopadhyay, and D. Doyle, "Damage assessment of CFRP composites using a time-frequency approach," Journal of Intelligent Material Systems and Structures, vol. 23, no. 4, pp. 397-413, 2012. 

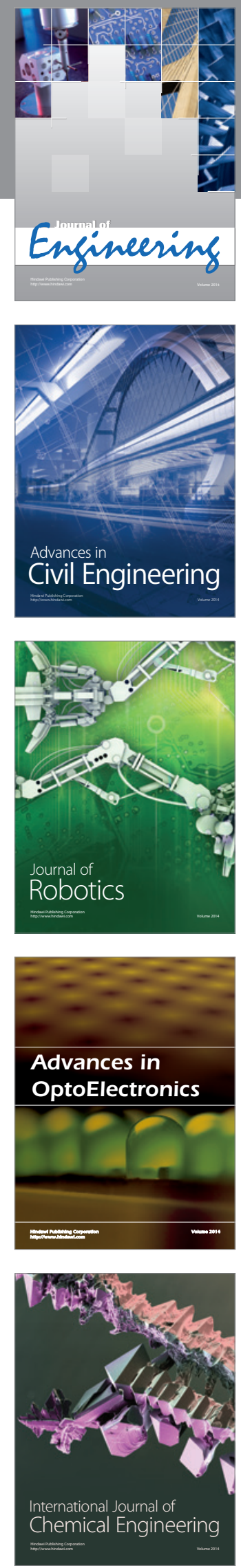

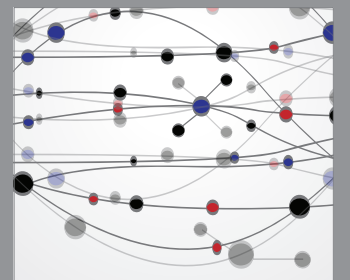

The Scientific World Journal
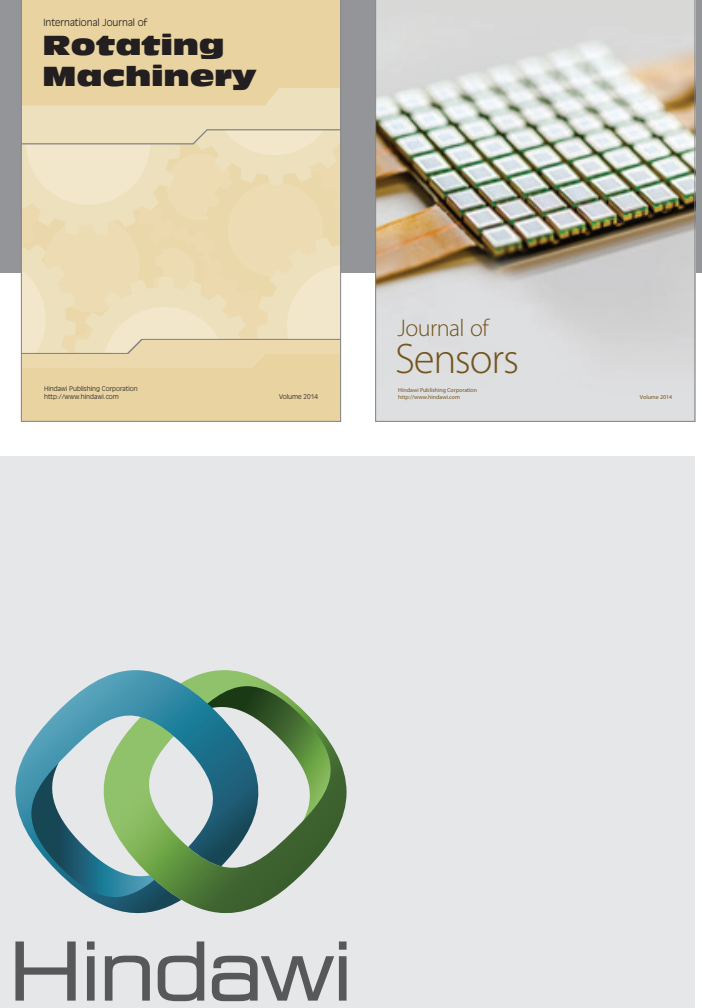

Submit your manuscripts at http://www.hindawi.com
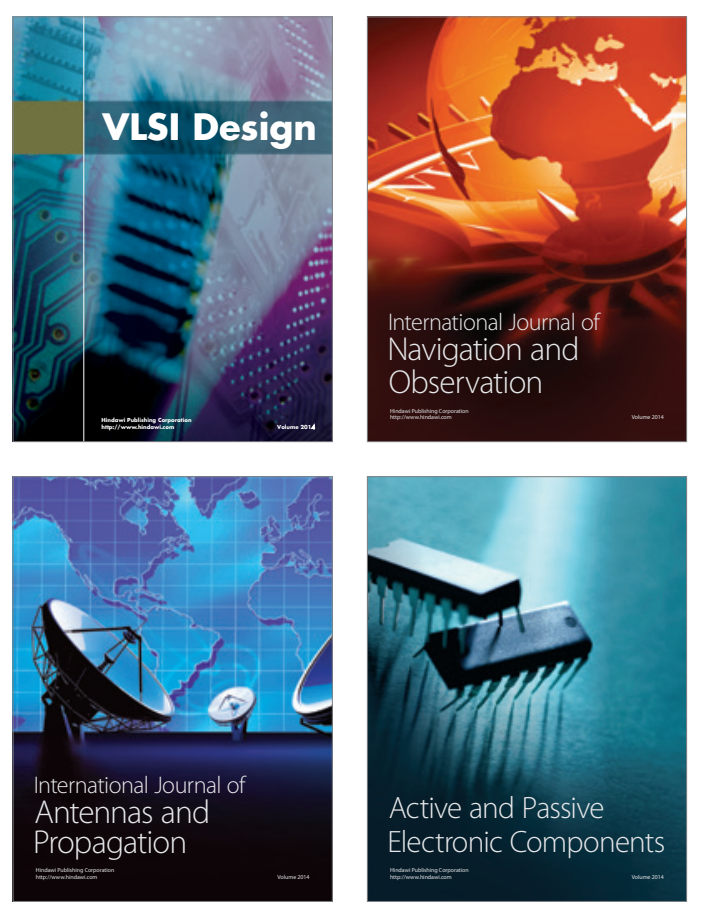
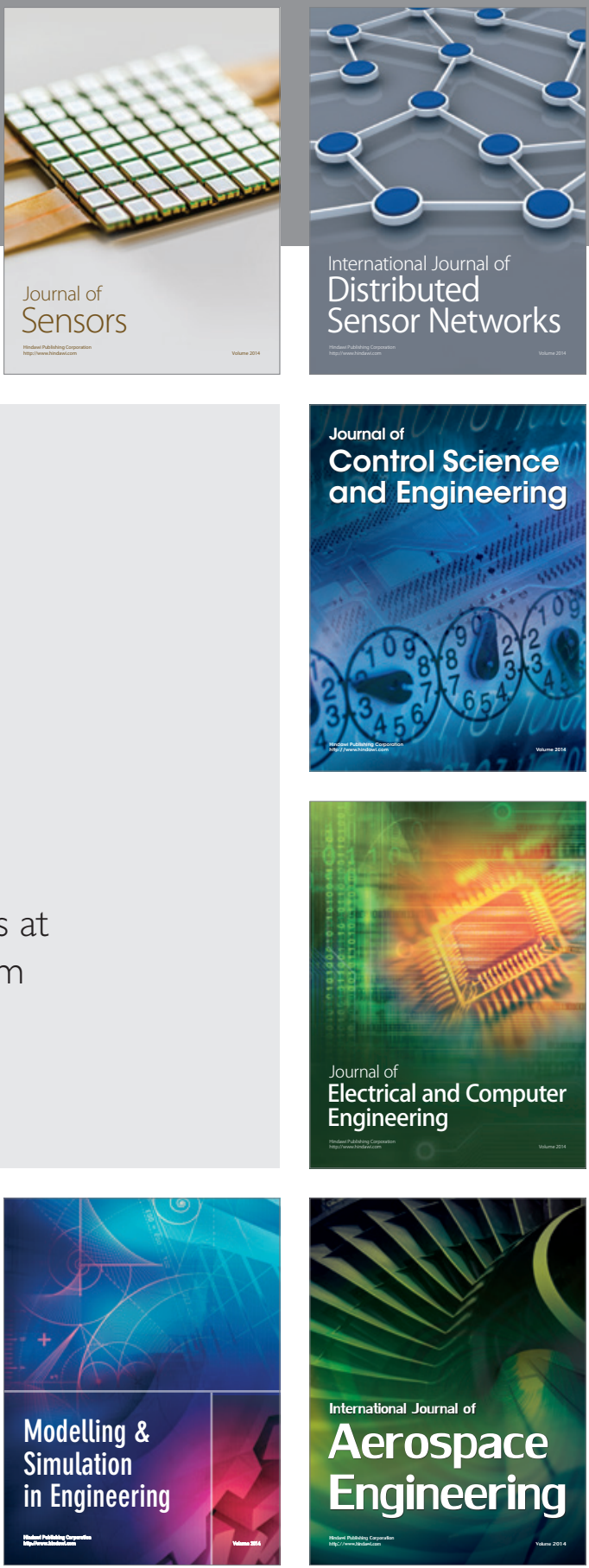

Journal of

Control Science

and Engineering
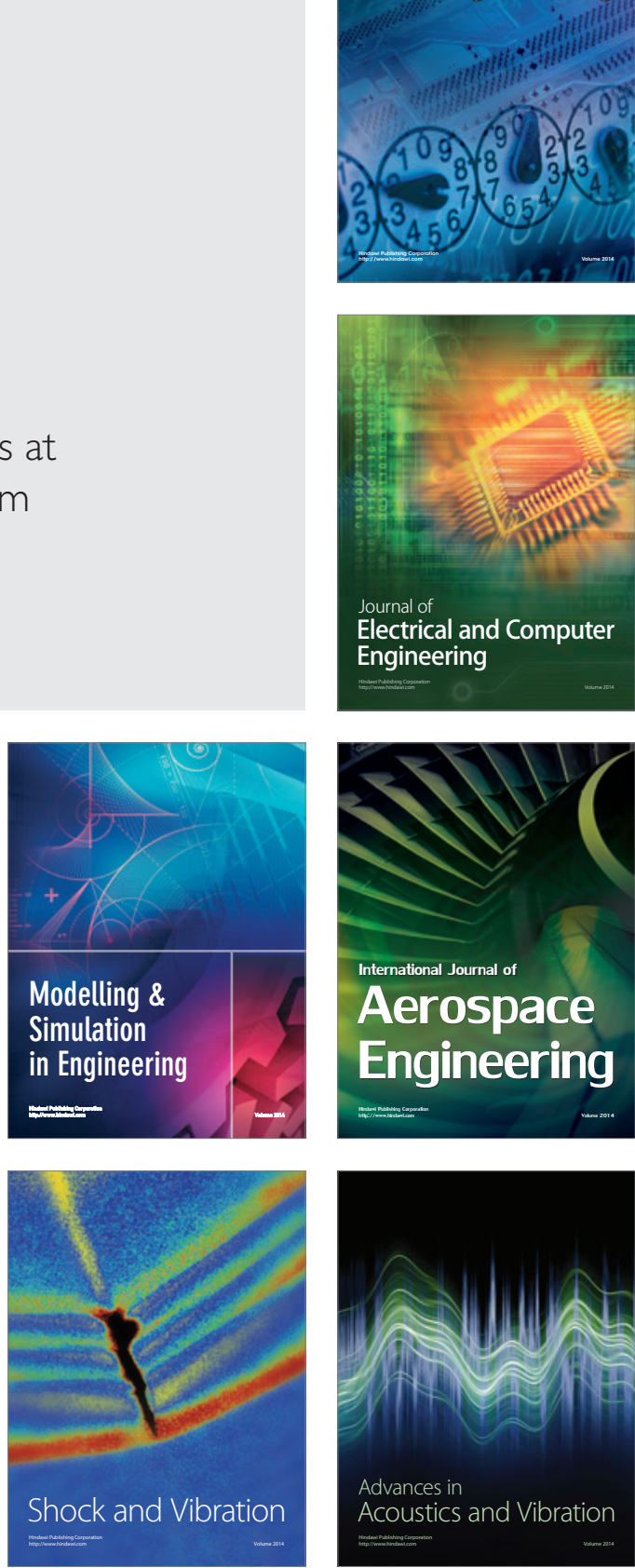